\title{
Musicoterapia: una alternativa de tratamiento para la ansiedad del personal de enfermería psiquiátrica
}

\author{
Gabriela Serna-Oviedo, * Sofía Cheverría Rivera**
}

\begin{abstract}
RESUMEN
La ansiedad es una patología psiquiátrica muy frecuente en México; afecta a personal de salud que labora en áreas de cuidados especiales, como la enfermería psiquiatría, por el contacto frecuente con enfermos cuyas patologías tienen manifestaciones conductuales. Generalmente es tratada con psicofármacos y psicoterapias, pero existen terapias alternativas con probada efectividad como la musicoterapia, aunque poco conocidas en el ámbito de la medicina convencional. Objetivo: Determinar el efecto de la musicoterapia en niveles de ansiedad del personal de enfermería de una clínica psiquiátrica de la ciudad de San Luis Potosí. Metodología: Estudio analítico, preexperimental; la población fue de 9 enfermeras seleccionadas por conveniencia, el instrumento de medición fue la escala autoaplicada para la evaluación de la ansiedad de Zung y la escala visual análoga de ansiedad; el plan terapéutico fue la aplicación del método Bonny en 6 sesiones. Se midió la ansiedad antes, durante y después de la terapia. Resultados: Predominó el género femenino (77.8\%); la edad promedio fue de 38 años y la desviación estándar de 2.9. En la medición previa, $66.7 \%$ calificó en rango normal de ansiedad, $22.2 \%$ moderada y $11.1 \%$ marcada; posterior a la terapia, $100 \%$ calificó en ansiedad normal. El promedio inicial fue 40.5 puntos ( $\mathrm{DE}=11.8)$ y el final de $28.1(\mathrm{DE}=7.7)$; en el análisis de diferencias se obtuvo $\mathrm{t}_{7}=3.58$ y $\mathrm{p}=0.004$. Conclusiones: La diferencia en puntos de ansiedad posterior a la terapia con música fue estadísticamente significativa, por lo que representa una alternativa en el tratamiento de ansiedad en personal de enfermería que atiende pacientes psiquiátricos.
\end{abstract}

Palabras clave: Ansiedad, enfermería psiquiátrica, musicoterapia.

\section{Music therapy: an alternative of treatment for the anxiety of the personnel of psychiatric infirmary}

\begin{abstract}
The anxiety is a very frequent psychiatric pathology in Mexico; affects health personnel that toils in areas of special cares, like the infirmary psychiatry, by the frequent contact with patients whose pathologies have behavioral manifestations. It is generally dealt with psico-drugs and psycotherapies, but alternative therapies with proven effectiveness like the music therapy exist, although little known in the scope of the conventional medicine. Objective: To determine the effect of the music therapy in levels of anxiety of the personnel of infirmary of a psychiatric clinic of the city of San Luis Potosí. Methodology: Analytical, pre-experimental study; the population was of 9 nurses selected by convenience, the measuring instrument was the Scale auto-aplicate for the evaluation of the anxiety of Zung and Analogous Visual Scale of Anxiety,
\end{abstract}

* Supervisora de Enfermería de Clínica Psiquiátrica "Dr. Everardo Neumann Peña".

** Docente de la Facultad de Enfermería de la UASLP.

Correspondencia: Gabriela Serna Oviedo. Km 8.5, Carretera a Matehuala, Soledad de Graciano Sánchez, S.L.P. Tel: (444) 831 20 23; (444) 83105 25, ext.33. E-mail: gabyserna_misamores@hotmail.com 
the therapeutic plan was the application of the Bonny method in 6 sessions, before was moderate the anxiety, during and after the therapy. Results: It predominated the female (77,8\%); the age average was of 38 years, standard deviation 2,9 ; in previous measurement $66,7 \%$ described in normal rank of anxiety, $22,2 \%$ moderate and $11,1 \%$ marked; subsequent to the therapy, $100 \%$ described in normal anxiety; the initial average was 40.5 points $(\mathrm{DE}=11.8)$ and end 28.1 and $(\mathrm{DE}=7.7)$, to the analysis of differences was obtained: $t_{7}=3.58$ and $p=0.004$. Conclusions: The difference in anxiety points subsequent to the therapy with music was statistically significant, reason why it represents an alternative in the treatment of anxiety in infirmary personnel that takes care of patients psychiatric.

Key words: Anxiety, psychiatric infirmary, music therapy.

\section{INTRODUCCIÓN}

$\mathrm{L}$ a ansiedad es una de las emociones más conocidas porque todas las personas la han sentido en algún momento de su vida. Es un proceso emocional ligado a la anticipación de las situaciones de peligro. Se le puede considerar como una actitud emocional cognitiva, o sea, un sistema de procesamiento de información amenazante que permite movilizar de forma anticipada acciones preventivas, ya que carece de recursos propios, los cuales toma prestados del miedo y el estrés, y opera como un programa de orden superior reclutando tales recursos sólo cuando le son precisos. ${ }^{1}$ Es una respuesta emocional frente a un estímulo indefinido o inespecífico, cuya diferencia entre normalidad y patología es difícil de establecer debido a que está muy relacionada con otros conceptos como el miedo, la angustia y el estrés; además de que también está considerada como una etiqueta diagnóstica para hacer referencia a una serie de trastornos, y es un término utilizado con frecuencia en el lenguaje coloquial de forma indiscriminada., ${ }^{1,2}$

La ansiedad normal y patológica tienen una misma raíz psicológica, ${ }^{1}$ pero la primera es un proceso emocional adaptativo e imprescindible para la vida, y la segunda es desproporcionada ante la situación que la desencadena, empeora el rendimiento, tiene mayor componente somático y genera la demanda de atención psiquiátrica. ${ }^{3}$

El proceso de ansiedad está determinado por el desarrollo personal, que ocasiona grandes diferencias en los elementos individuales e interactúa con otros rasgos de personalidad, ambientales y hereditarios, los cuales originan mayor susceptibilidad y vulnerabilidad para padecerla. ${ }^{1}$

La ansiedad está considerada como el principal problema de salud en los ámbitos internacional, nacional y estatal. En el internacional, los estudios encontrados hacen referencia, en su mayoría, a datos encontrados en población general que padece algún trastorno de ansiedad, y coinciden en que la prevalencia de los trastornos de ansiedad en la población en general es mayor a $10 \%$.

Otros estudios realizados en Estados Unidos muestran que hasta tres cuartos de la población tiene uno o más miedos irracionales, pánico repentino, o bien son personas nerviosas. ${ }^{4}$ En México, los últimos estudios epidemiológicos realizados en atención primaria reflejan una mayor utilización de servicios médicos por pacientes con síntomas de ansiedad en relación con otros pacientes, en especial aquellos que presentan síntomas somáticos cardiovasculares, que requieren de tratamientos costosos con el consecuente aumento en los gastos de las instituciones, y el mal diagnóstico de ansiedad. ${ }^{3}$ Aún no se han encontrado estudios realizados específicamente con personal de enfermería psiquiátrica; los revisados hacen referencia a que el personal de salud es uno de los más afectados por la ansiedad, ${ }^{4-9}$ sobre todo el de enfermería, en particular en áreas de cuidados especiales o críticos, ya que desempeñarse en estas áreas provoca muchas tensiones emocionales por el hecho de enfrentarse a la muerte y sufrimiento, además de a factores laborales como la división técnica, jerárquica y social del trabajo.

Entre las áreas especiales, la enfermería psiquiátrica, considerada como un proceso que lleva a una relación interpersonal estrecha, ${ }^{10,11}$ es una de las más afectadas, porque las enfermedades mentales se manifiestan en conductas como la agresiva, la hostil, la dependiente, la suspicaz, la maníaca, la depresiva y la suicida, lo cual influye en extremo en la salud física y mental de este personal.

La constante ansiedad de este personal tiene consecuencias en todos los ámbitos: en el laboral, inadecuadas relaciones interpersonales, falta de motivación, con disminución en el desempeño y la productividad, y el ausentismo, que conduce a una sobrecarga de trabajo para el personal que sí asiste. Todo ello afecta la calidad de la atención que se brinda al usuario e incrementa los gastos institucionales; en el familiar y en el social, relaciones inadecuadas y conflictivas.

Las consecuencias más graves, compuestas por un amplio elenco de trastornos, no se deben tanto a un mal funcionamiento del propio proceso, sino más bien a un afrontamiento inadecuado. ${ }^{1}$ Además, los trastornos de ansiedad, una vez presentes, tienden a la cronicidad y a complicarse con la aparición de nuevos trastornos,${ }^{12}$ como los de pánico, obsesivo compulsivos y de ansiedad acompañada de alguna fobia. 
Por la importancia de esta emoción y por el hecho de que una vez que se ha manifestado como trastorno es tratada generalmente con psicofármacos y psicoterapias en sus diversas modalidades, además de ser un problema con frecuencia subdiagnosticado, se hace necesario tomar medidas preventivas que eviten que pase de una ansiedad normal a la patológica, para lo cual existe una diversidad de tratamientos, siendo uno de ellos la musicoterapia.

La musicoterapia es definida por la National Association for Music Therapy (NAMT), en 1980, como el uso de la música en la consecución de objetivos terapéuticos, como son la restauración, el mantenimiento de la salud tanto física como mental. Es también la aplicación científica de la música, dirigida por el terapeuta en un contexto terapéutico para provocar cambios en el comportamiento; dichos cambios facilitan a la persona el tratamiento que debe recibir, a fin de que pueda comprenderse mejor a sí misma y a su mundo, para poder ajustarse mejor y más adecuadamente a la sociedad. ${ }^{13}$ Comprende toda terapia que utilice las propiedades del sonido y la vibración como método de curación o alivio para gran parte de los males psicofísicos que padece el ser humano. ${ }^{14}$ Los componentes básicos de la música, como ritmo, melodía y armonía, son los mismos que componen a nuestro organismo, el ritmo cardiaco, la sincronización rítmica al caminar, la melodía y volumen de la voz al hablar. ${ }^{15}$

Como técnica terapéutica, el uso de la música no es nada nuevo, las sociedades primitivas desde la prehistoria, y las existentes, han utilizado cantos, danzas e instrumentos musicales muy rudimentarios en los rituales religiosos o sociales. El sonido siempre se ha considerado un enlace directo entre la humanidad y lo divino; en cierta forma, toda la escuela antigua del misterio enseñó a sus estudiantes el uso del sonido como fuerza creativa y curativa. La música se considera la más vieja forma de cura, y antiguamente era una parte predominante de la enseñanza temprana de los griegos, los chinos, los indios del este, los tibetanos, los egipcios, los indios americanos, los mayas y los aztecas. ${ }^{16}$ En la antigua Grecia se experimentaba con los enfermos, sometiéndolos a diversos tipos de música. Hipócrates la utilizaba con los enfermos de la mente y Aristóteles señaló la importancia de ésta ante las emociones incontrolables, explicó el efecto beneficioso para motivar la catarsis; mientras Platón recomendaba la danza y la música a personas que padecían angustia fóbica y miedo o terror. ${ }^{17}$

Los chamanes o curanderos de los pueblos primitivos, y posteriormente los médicos de las civilizaciones más desarrolladas, han sido los encargados de explicar y desarrollar las terapias musicales a lo largo de los siglos en las diferentes culturas. En el siglo XIX se encuentran datos en revistas y libros, tanto de música como de medicina; todos apoyaban la música como alternativa o complemento al tratamiento médico tradicional. ${ }^{17}$

La concepción de la música como material emotivo, potenciador de sentimientos, desarrollada con la doctrina de los afectos, va a estimular de manera decisiva la mentalización social sobre las posibilidades terapéuticas de la música, sobre todo en el ámbito de las enfermedades del estado de ánimo, específicamente en estados depresivos con inhibición psicomotora y estados de agitación y ansiedad. ${ }^{17}$

En enfermería, la utilización de la música se inició con Florence Nightingale, y más tarde fue también utilizada con los heridos de la Segunda Guerra Mundial, así como en varias otras situaciones clínicas, como control del dolor e inducción al relajamiento, con lo que se obtuvieron buenos resultados. ${ }^{18}$ En la actualidad, la musicoterapia tiene un carácter científico, ya que según la física, la vida está compuesta de átomos que contienen protones y electrones, los cuales se encuentran cargados de energía y en movimiento constante, siendo sus movimientos audibles, las vibraciones sanas de las que habla la física están conectadas con las vibraciones y las ondulaciones de átomos y de moléculas dentro del aire. En prácticas curativas, el ritmo puede energizar y estimular nuestras energías básicas principales; con la percusión se activan los centros chakra del bazo y de la base del cuerpo, relacionados con el sistema circulatorio de las glándulas suprarrenales, y de nuestra fuerza básica de la vida, así como de la sexualidad y nuestra vitalidad espiritual dinámica. Otra parte de la música, que es la armonía, puede ligar nuestra energía individual con la energía de lo divino; tiene efecto en el aspecto físico, emocional y mental, pero sobre todo en el espiritual. ${ }^{16}$

El sonido en cualquiera de sus formas es una fuente de energía; si el desequilibrio ha ocurrido dentro de los parámetros electromagnéticos normales de los cuerpos, se puede utilizar el sonido para ayudar a restaurar la homeostasis, aliviar el dolor o acelerar la cura; facilita también la concentración, la relajación, el aprendizaje, la creatividad y un conocimiento creciente de estados psicoespirituales. ${ }^{16}$

Los tonos, los ritmos, los instrumentos y las vocalizaciones específicas se pueden utilizar para estimular y balancear el flujo de energía de los chakras, lo que actuará sobre los órganos y los sistemas físicos; es decir, en muchos casos, simplemente al restablecer el equilibrio y el funcionamiento de los chakras, el cuerpo puede responder más fácilmente a las manifestaciones físicas de la enfermedad. ${ }^{16}$

Existen profesionales dedicados a su aplicación; en los principales países de Europa la musicoterapia está bastante extendida en la teoría y en la praxis, aunque su incorporación al mundo universitario data de hace unos 40 años. Como disciplina de salud la musicoterapia se ha extendido alrededor del mundo; se han desarrollado programas de grado y 
postgrado en Europa (Alemania, Austria, Dinamarca, Italia, Francia, Hungría, Polonia, Reino Unido, España e Israel), en Asia y Oceanía (Korea, Taiwán, Finlandia y Australia), y en el continente americano (Estados Unidos, Canadá, Brasil, Cuba, Puerto Rico, Venezuela, Uruguay, Perú, Argentina, México y Chile). ${ }^{19}$

Según sus efectos se le clasifica en música sedante, la cual tiene un ritmo regular, una dinámica predecible, consonancia armónica y un timbre vocal e instrumental reconocido, con efectos tranquilizantes, y en estimulante, que aumenta la energía corporal, induce a la acción y estimula las emociones. A través de la escucha o la creación, la música imprime una energía de carácter global que circula libremente en el interior de la persona para proyectarse después a través de las múltiples vías de expresión disponibles. Ésta, al igual que otros estímulos portadores de energía, produce un amplio abanico de respuestas que pueden ser inmediatas, diferidas, voluntarias o involuntarias, que dependen de las circunstancias personales (edad, etapa del desarrollo, estado anímico, salud psicológica, apetencia).$^{19}$

Se ha demostrado también cómo la música puede hacer disminuir el dolor, la ansiedad y el estrés; su uso puede beneficiar a quienes buscan un desarrollo personal, a quienes padecen trastornos mentales, personas con discapacidades físicas o sensoriales, personas de la tercera edad, niños con problemas de aprendizaje o trastornos del lenguaje, mujeres embarazadas, pacientes en situación terminal, a quienes se encuentran en estado de coma o a quienes padecen de adicciones o se encuentran internos en cárceles. ${ }^{17}$

Existen estudios que nos hablan sobre la efectividad de la musicoterapia, tal es el caso del de Aparicio J y colaboradores, quienes en el 2004 realizaron un estudio con 50 pacientes postoperados, aplicando previamente el cuestionario Escala de Ansiedad Estado-Rasgo (STAI) al ingreso en la unidad hospitalaria, 25 de los cuales fueron sometidos a terapia musical como parte de los cuidados de enfermería, encontrando que las mujeres presentan un mayor nivel de ansiedad que los hombres, tanto antes como después de la intervención con o sin musicoterapia; se encontró que la ansiedad disminuye notablemente, sea cual sea el tipo de anestesia que se aplique. ${ }^{20}$

Flores-Gutiérrez E y Díaz J, en 2009, llevaron a cabo un estudio que tuvo como objetivo ver la respuesta emocional a la música y la atribución de términos de la emoción a piezas musicales seleccionadas en referencia a los estados de ánimo que su audición provoca en voluntarios sanos. Encontraron que con la pieza musical de Mozart la respuesta afectiva se inclinaba hacia términos agradables y estimulantes. ${ }^{21}$

La terapia del sonido se relaciona con las demás ciencias de la salud como: medicina, fisioterapia, logopedia, terapia ocupacional y psicología social. La educación musical cum- plía una labor muy importante en los inicios de la terapia del sonido, en su mayoría eran pedagogos musicales los que empezaron a trabajar con esta terapia enfocada a seres discapacitados, para ayudarles a tener una mejor calidad de vida; Edgar Willems y otros desarrollaron métodos de enseñanza de música que transformaron los métodos tradicionales. ${ }^{17}$ El método Bonny de imagen guiada y música ${ }^{17}$ es uno de los más utilizados en musicoterapia, ya que brinda una mayor relajación en el paciente: favorece la disminución de la ansiedad, se identifica por el uso de programas de música clásica, específicamente elegida dentro de una sesión de uno a uno o en series de sesiones dirigidas por un facilitador formado en este método; estas sesiones están caracterizadas por una progresión pautada de componentes que dan forma, continuidad y sensación de seguridad al proceso terapéutico.

Es una terapia integrativa y asistida por música que facilita las exploraciones de la consciencia que pueden llevar a la transformación e integridad. Se desarrolló como método a través de la investigación y práctica por la Dra. Helen L. Bonny. En este método las exploraciones de la consciencia son viajes a través de experiencias sensoriales, biográficas, somáticas, simbólicas y de imágenes arquetípicas activadas musicalmente, las cuales expanden la percepción individual de integridad afirmando el sentido de conexión con las realidades que trascienden lo personal.

Requiere de un formador facilitador que refine la intuición, la apertura, las habilidades clínicas, la consciencia musical, la ética y la espiritualidad, con el fin de responder a las dinámicas de estas exploraciones, así como proporcionar el contexto en el que se puede dar la integración de experiencias interiores complejas.

Una sesión en el método Bonny requiere de la siguiente preparación:

1. Una discusión preliminar entre un facilitador y un cliente puede incluir información biográfica, establecimiento de objetivos y sentimientos o preocupaciones actuales.

2. El facilitador proporciona sugerencias verbales para relajar el cuerpo y centrar la mente del paciente, favoreciendo así la entrada a un estado de consciencia alterado.

3. El facilitador escoge un programa musical apropiado a la disposición del paciente para seguir el proceso terapéutico.

\section{Experiencia musical interactiva:}

1. El paciente escucha la música y expresa las imágenes evocadas por ella.

2. El facilitador interactúa verbalmente con el cliente, de forma que apoya y realza las experiencias musicales y de imágenes del cliente. 


\section{Cierre}

Al final del programa musical el facilitador ayuda al paciente a volver a un estado de vigilia de la consciencia.

\section{Integración}

La integración de las experiencias de las sesiones continúa tanto a través de la discusión reflexiva y de las actividades expresivas realizadas inmediatamente después de la música y durante todo el proceso terapéutico.

De esta forma, el presente trabajo tiene como propósito ofrecer al personal que realiza funciones de enfermería psiquiátrica y a las autoridades competentes de la Clínica Psiquiátrica "Dr. Everardo Neumann Peña", nuevas alternativas en el manejo de la ansiedad, e incluirlas como parte de un programa de desarrollo para el personal, partiendo de la experiencia de que en el ámbito de la salud mental y la psiquiatría, efectivamente el personal de enfermería se encuentra expuesto a la ansiedad constante y del hecho que en la clínica en mención se conoce la presencia de algunos desórdenes emocionales como ansiedad y depresión en algunos compañeros de enfermería, razón por la cual surge el interés por parte de la investigadora de realizar el presente estudio, con el fin de conocer cuál es el efecto de la musicoterapia en los niveles de ansiedad.

Para lo anterior, se midió la ansiedad del personal de enfermería a través de la escala autoaplicada para la evaluación de la ansiedad de Zung, previo consentimiento informado, y se implementó el tratamiento con música, con base en un plan establecido, realizando la evaluación de las respuestas dadas en cada participante mediante la aplicación de la escala visual análoga de ansiedad y la propia escala de Zung, con lo que finalmente se comprobó la efectividad del tratamiento en la disminución de los niveles de ansiedad del personal de enfermería psiquiátrica.

Se realizó un estudio analítico cuasiexperimental. La población estuvo conformada por nueve enfermeros que laboraban en una clínica psiquiátrica de San Luis Potosí y que según la escala autoaplicada de ansiedad de Zung, obtuvieron de 20 a 44 puntos.

\section{MATERIAL Y MÉTODOS}

Es un estudio analítico, preexperimental, prospectivo, con muestreo no probabilístico por conveniencia, en el cual participaron nueve miembros del personal de enfermería de una clínica psiquiátrica de San Luis Potosí.

Se consideraron los siguientes criterios de inclusión:

- Personal de enfermería psiquiátrica: con antigüedad laboral mínima de un año en la clínica psiquiátrica.
- Terapeuta: certificado en musicoterapia, con disponibilidad de tiempo y que aplique en su ejercicio los principios de ética establecidos para esta terapia. Que den su consentimiento informado por escrito y que tengan disponibilidad de tiempo para acudir a las terapias en los horarios programados.

Criterio de exclusión: las personas que al momento de la aplicación del instrumento se encuentren recibiendo terapia con energía o tratamiento médico para la ansiedad.

Criterio de eliminación: quienes, una vez iniciadas las terapias, decidan de manera voluntaria retirarse del estudio; aquellos participantes a los que no se les aplique el total de las sesiones de terapia programadas o que cambien de trabajo o área de adscripción.

La evaluación de los niveles de ansiedad se realizó en las instalaciones de la Clínica Psiquiátrica Dr. Everardo Neumann Peña, ubicada en el kilómetro 8.5 de la carretera a Matehuala, en Soledad de Graciano Sánchez, del 19 al 24 de mayo de 2009, utilizando la escala autoaplicada para la evaluación de la ansiedad de Zung, ${ }^{22-24}$ la cual tiene aceptables propiedades psicométricas y es útil para identificar posibles casos en forma rápida y económica. Consta de 20 preguntas, 15 para síntomas somáticos y 5 para cognitivos, conductuales o afectivos, las cuales hacen referencia a un periodo anterior de 30 días; cada pregunta puntúa de uno a cuatro; $25 \%$ planteadas en sentido positivo y $75 \%$ en sentido inverso; el puntaje es de 20 a 80 puntos, y para contestar se requiere de 10 minutos aproximadamente.

La aplicación de la terapia con música utilizó el método Bonny; constó de seis sesiones con duración de dos horas cada una, dos veces por semana. Se llevó a cabo en la sala de usos múltiples de la Unidad de Postgrado de la Facultad de Enfermería de la UASLP, de junio a julio de 2009. Antes y después de cada sesión se aplicó a los participantes la escala visual análoga de ansiedad, lo que nos permitió conocer las respuestas inmediatas ante el tratamiento; esta escala es un instrumento de tipo gráfico que utiliza representaciones visuales concretas; válido y confiable en la evaluación de la ansiedad, además de que ha sido muy utilizada en adultos..$^{25,26}$

Los datos se capturaron, organizaron y procesaron en el programa estadístico para las ciencias sociales SPSS versión 15. Se aplicó estadística descriptiva, frecuencias, medidas de tendencia central y de dispersión. Para probar la hipótesis se aplicó t de Student pareada. La presentación de los datos se realizó en tablas cruzadas de los niveles de antes y después de la aplicación de la terapia.

La presente investigación se inscribió en la línea de investigación denominada Gestión e Innovación del Cuidado de Enfermería. Para su revisión, se presentó al Comité de Ética 
e Investigación de la Facultad de Enfermería de la UASLP, y fue aprobada y registrada con CLAVE: 0020HCEI-ENF09, con fecha 30 de enero de 2009, así como por parte del Comité de Enseñanza, Investigación y Ética de la institución en la cual se llevó a cabo. Para su aplicación se basó en el Reglamento de la Ley General de Salud en Materia de Investigación, el Código de Ética para enfermeras y enfermeros en México y el Código de Ética para musicoterapeutas.

\section{RESULTADOS}

La población de estudio estuvo conformado por nueve participantes, predominó el sexo femenino con $77.8 \%$, la edad promedio fue de 38 años (DE de 2.9), el rango de edad predominante fue de 35 a 39 años; casados 66.6\%; escolaridad a nivel técnico 77.8\%, como lo muestra el cuadro $I$. En cuanto a las características laborales, $55.6 \%$ tenía de 11 a 15 años de antigüedad, el mismo porcentaje laboraba en el turno de jornada acumulada y tenía código de camillero, $66.6 \%$ asignados a sala de mujeres y 44.4 contaba con trabajo alterno, como se observa en el cuadro II.

En el cuadro III se observan los niveles de ansiedad obtenidos antes, durante y después de la aplicación de la terapia con música; en la medición inicial, $66.7 \%$ se encontró en rango normal de ansiedad, $22.2 \%$ en moderada y 11.1 en marcada; en la evaluación intermedia, aplicada después de la tercera sesión, $88.9 \%$ se encontró en el rango normal, y al concluir el tratamiento el $100 \%$ de los participantes.

El promedio de puntos de ansiedad obtenido con la escala de Zung fue de 40.5 (DE 11.8) en la medición inicial, en la intermedia de 32.1 (DE 9.7) y al final de 28.1 (DE de 7.7), con lo que se obtuvo un promedio de diferencias estadísticamen-

Cuadro I. Características sociodemográficas del personal de enfermería psiquiátrica, San Luis Potosí, mayo 2009.

\begin{tabular}{lcc}
\hline Características & número & $\begin{array}{c}\mathrm{n}=9 \\
\%\end{array}$ \\
\hline Edad & 1 & 11 \\
$\quad 30$ a 34 & 5 & 55.5 \\
35 a 39 & 3 & 33.5 \\
$\quad 40$ y más & & \\
Sexo & 7 & 77.8 \\
$\quad$ Femenino & 2 & 22.2 \\
$\quad$ Masculino & 2 & 22.2 \\
Estado civil & 2 & 66.6 \\
$\quad$ Soltero & 6 & 11.1 \\
$\quad$ Casado & 1 & 77.8 \\
$\quad$ Madre soltera & & 11.1 \\
Escolaridad & 7 & 11.1 \\
$\quad$ Précnica & 1 & \\
$\quad$ Postgrado & 1 & \\
\hline
\end{tabular}

Fuente: Escala autoaplicada para la evaluación de la ansiedad de Zung. te significativo con una $\mathrm{t}_{7}=3.58 \mathrm{y} \mathrm{p}=0.004$ (Cuadro IV). Con la aplicación de la escala visual análoga de ansiedad, el porcentaje promedio de ansiedad percibido por los participantes antes de recibir la terapia fue de $47.3 \%$ (DE 33.8\%), y al concluir la misma de $0.07 \%$ (DE 0.27 ), con un promedio de diferencias estadísticamente significativo, al obtener $\mathrm{t}_{7}=$ 3.59 y $\mathrm{p}=0.004$, según lo observado en el cuadro $\mathrm{V}$.

$\mathrm{Al}$ analizar las características de los participantes que calificaron en nivel moderado de ansiedad, siendo $22.2 \%$ de la población estudiada, se encontró que la totalidad era de sexo femenino, contaba con un trabajo alterno y tenía laborando en la institución entre 11 y 15 años; $11.1 \%$ solteros y $11.1 \%$ casados desempeñándose como enfermeros encargados de servicio; $11.1 \%$ contaba con carrera técnica y en el mismo porcentaje eran profesionistas; en igualdad de porcentajes, laboran en turno nocturno y jornada acumulada, asignados a los servicios de sala B (hombres de mediana estancia) y sala C (mujeres de mediana y larga estancia). En lo referente al $11.1 \%$ que calificó en nivel de ansiedad marcada o severa, de sexo masculino, con trabajo alterno y antigüedad laboral entre 16 y 20 años, casado, con puesto de encargado de servicio, formación académica a nivel técnico, laborando en el turno de jornada acumulada, con asignación en la sala $\mathrm{C}$ (mujeres de mediana y larga estancia).

\section{DISCUSIÓN}

Respecto a las características de la población estudiada, en las cuales se observa que $\mathbf{7 7 . 8 \%}$ lo representó el sexo

Cuadro II. Características laborales del personal de enfermería psiquiátrica San Luis Potosí, mayo 2009.

\begin{tabular}{|c|c|c|}
\hline Características & número & $\begin{array}{c}\mathrm{n}=9 \\
\%\end{array}$ \\
\hline \multicolumn{3}{|l|}{ Antigüedad laboral/años } \\
\hline 1 a 5 & 1 & 11.1 \\
\hline 6 a 10 & 1 & 11.1 \\
\hline 11 a 15 & 5 & 55.6 \\
\hline 16 a 20 & 2 & 22.2 \\
\hline \multicolumn{3}{|l|}{ Turno } \\
\hline Matutino & 1 & 1.1 \\
\hline Nocturno & 3 & 3.3 \\
\hline Jornada acumulada & 5 & 55.6 \\
\hline \multicolumn{3}{|l|}{ Puesto } \\
\hline Camillero & 5 & 55.6 \\
\hline Enfermera encargada de servicio & 3 & 33.3 \\
\hline Supervisora & 1 & 11.1 \\
\hline \multicolumn{3}{|l|}{ Servicio } \\
\hline Sala B & 1 & 11.1 \\
\hline Sala C & 6 & 66.7 \\
\hline Sala G & 1 & 11.1 \\
\hline Supervisión & 1 & 11.1 \\
\hline \multicolumn{3}{|l|}{ Trabajo alterno } \\
\hline Sí & 4 & 44.4 \\
\hline No & 5 & 55.6 \\
\hline
\end{tabular}

Fuente: Escala autoaplicada para la evaluación de la ansiedad de Zung. 
Cuadro III. Nivel de ansiedad del personal de enfermería psiquiátrica, San Luis Potosí, mayo-julio 2009.

\begin{tabular}{|c|c|c|c|c|c|c|}
\hline \multirow{3}{*}{ Nivel de ansiedad } & \multicolumn{6}{|c|}{$\mathrm{n}=9$} \\
\hline & \multicolumn{2}{|c|}{ Inicial } & \multicolumn{2}{|c|}{ Intermedia } & \multicolumn{2}{|c|}{ Final } \\
\hline & No. & $\%$ & No. & $\%$ & No. & $\%$ \\
\hline Rango normal & 6 & 66.7 & 8 & 88.9 & 9 & 100 \\
\hline Moderada & 2 & 22.2 & 1 & 11.1 & 0 & 0 \\
\hline Marcada o severa & 1 & 11.1 & 0 & 0 & 0 & 0 \\
\hline Extrema & 0 & 0 & 0 & 0 & 0 & 0 \\
\hline Total & 9 & 100 & 9 & 100 & 9 & 100 \\
\hline
\end{tabular}

Fuente: Escala autoaplicada para la evaluación de la ansiedad de Zung.

Cuadro IV. Puntaje promedio de ansiedad obtenido antes, durante y después de la musicoterapia, San Luis Potosí, mayo-julio 2009.

\begin{tabular}{|c|c|c|c|c|c|}
\hline Evaluación & Promedio & Desviación estándar & Mínimo & Mínimo & $\begin{array}{c}n=9 \\
\text { Significancia }\end{array}$ \\
\hline Inicial & 40.5 & 11.8 & 23 & 61 & $\mathrm{~T} 7=3.58$ \\
\hline Intermedia & 32.1 & 9.7 & 20 & 52 & Pt7 $=0.004$ \\
\hline Final & 28.1 & 7.7 & 20 & 42 & \\
\hline
\end{tabular}

Fuente: Escala autoaplicada para la evaluación de la ansiedad de Zung.

Cuadro V. Porcentaje promedio de ansiedad obtenido antes, durante y después de la aplicación de la terapia con música, San Luis Potosí, mayo-julio 2009.

\begin{tabular}{|c|c|c|c|c|c|}
\hline Evaluación & Promedio & Desviación estándar & Mínimo & Máximo & $\begin{array}{c}n=9 \\
\text { Significancia }\end{array}$ \\
\hline Inicial & 70.4 & 23.6 & 33 & 98 & $\mathrm{~T} 7=3.59$ \\
\hline Posterior a la tercera sesión & 24.2 & 17.5 & 0 & 58 & $\mathrm{Pt7}=0.004$ \\
\hline Final & 8.7 & 10.8 & 0 & 27 & \\
\hline
\end{tabular}

Fuente: Escala Visual Análoga de Ansiedad.

femenino, con una edad promedio de 38.1 años, coinciden con los datos internacionales registrados por la Epidemiologyc Catchmen Area (ECA), referidos por Stein D en el 2005, y por la guía práctica Manejo del espectro depresiónansiedad 2004, los cuales revelaron que los trastornos de ansiedad constituyen uno de los grupos más prevalentes en los Estados Unidos, con mayor frecuencia en las mujeres en una proporción de 2 a 1 y de inicio en edades tempranas; también, que tres cuartas partes de la población tienen uno o más miedos irracionales, pánico repentino, o bien son personas "nerviosas". A nivel nacional, la depresión y la ansiedad son las psicopatologías más frecuentes; la Encuesta Nacional de Enfermedades Psiquiátricas reportó que el $14.3 \%$ de la población encuestada había sufrido alguna vez en su vida algún tipo de trastorno de ansiedad: $18.5 \%$ de las mujeres y $9.5 \%$ de los hombres. ${ }^{5,27}$

Si bien el número de población estudiada fue pequeño, es importante resaltar que el hecho de que la mayoría de quienes aceptaron participar en el proyecto fueran mujeres es indicativo de la conciencia que tienen las enfermeras acerca de que la profesión que profesa es eminentemente femenina, que como mujer está expuesta a una diversidad de roles s $^{3,28}$ que la hacen aún más vulnerable de padecer alteraciones físicas y mentales, y a lo mencionado por Valderrama en su estudio, en el que considera que la mujer expresa más sus sentimientos, situación que le permite solicitar ayuda y responder de manera más adecuada a los tratamientos establecidos. ${ }^{29}$

En el análisis de las diferencias se encontró que la terapia con música fue efectiva, con una significancia de $\mathrm{p}=$ 0.004 según la escala de Zung y la escala visual análoga de ansiedad. Lo anterior se respalda con lo que refiere la teoría acerca del efecto tranquilizante y relajante que tiene la música en el ser humano, así como por lo referido por García-Viniegras y colaboradores en su estudio $\mathrm{Mu}$ sicoterapia. Una modalidad terapéutica para el estrés laboral, en el que menciona que en su centro de atención al estrés, con frecuencia han aplicado la música relajante, la antidepresiva y la ansiolítica, con resultados altamente positivos. ${ }^{30}$ Trallero C por su parte, en el estudio Tratamiento del estrés docente y prevención del Burnout con musicoterapia autorrealizadora, encontró la ansiedad 
como síntoma de Burnout en $80.33 \%$; en el estudio se aplicaron 16 sesiones y tenían como objetivo comprobar la eficacia del tratamiento un mes después de la última sesión y habiendo terminado el curso, y constatar si los efectos conseguidos eran duraderos; se obtuvo que este tratamiento disminuyó de manera considerable la ansiedad rasgo y la ansiedad estado. ${ }^{31}$

Aparicio $\mathrm{M}$ y asociados encontraron que la ansiedad del paciente medicoquirúrgico sometido a una operación, en el postoperatorio inmediato disminuye notablemente al recibir musicoterapia, sea cual sea el tipo de anestesia recibida. ${ }^{20}$

Al comparar la respuesta ante la musicoterapia por sexo, llama la atención que de los dos participantes del sexo masculino, uno tuvo buena respuesta, aunque menor en relación a las de las participantes del sexo femenino, y el segundo incluso aumentó dos puntos en la escala de Zung al final del tratamiento, coincidente con lo que Valderrama menciona acerca de que los hombres no son tan sensibles ante su mundo emocional y presentan dificultad para expresar sus sentimientos, por ello no se pueden relajar o les cuesta trabajo hacerlo, lo que puede llevarlos incluso a aumentar su estado de ansiedad; en cambio, en las mujeres es menor la resistencia ante la expresión de sus sentimientos, lo que se traduciría en un decremento del nivel del estado de ansiedad. ${ }^{29}$

\section{CONCLUSIONES}

Con base en los resultados obtenidos se llega a las siguientes conclusiones:

- La población estudiada es un grupo homogéneo en cuanto a las características socio-demográficas y laborales.

- Aun cuando la mayoría de los participantes calificó en el rango normal de ansiedad, el hecho de encontrar en algunos de ellos niveles moderado y marcado de ansiedad es indicativo de que este personal se encuentra en eminente riesgo de avanzar hacia un estado patológico.

- La terapia con música resultó efectiva para el tratamiento de la ansiedad en personal de enfermería psiquiátrica, por lo que habría que comparar los resultados de este estudio con otros de ansiedad en personal de características semejantes.

\section{Limitantes:}

- La falta de diversificación de horarios para la aplicación de la terapia impidió la participación de algunas personas interesadas, quienes se encontraban laborando en el turno vespertino, o bien rotando turnos.

- La presencia de distractores en algunos de los lugares en los cuales se implementaron algunas sesiones.

\section{RECOMENDACIONES}

Para la Clínica Psiquiátrica Dr. Everardo Neumann Peña se debe de implementar un programa de desarrollo humano que permita adoptar esta terapia de manera sistemática en el personal de enfermería y el resto de las disciplinas, en función de que diversos estudios reportan su efectividad y el presente no es la excepción. Considerando que el tratamiento no únicamente mejora la ansiedad, sino que, secundario a ello, se daría un aumento en el desempeño, productividad y en las relaciones humanas, lo cual se vería reflejado en la atención que se brinda al usuario y en la disminución de costos para la institución.

Fomentar la aplicación de estas terapias como complementarias en la atención a los usuarios que atiende el personal de salud, para quienes estén interesados en continuar la investigación respecto a esta temática.

Realizar otros estudios dirigidos al personal de salud, en especial al de enfermería psiquiátrica, considerando un mayor número de participantes; también se deben considerar diversos horarios para la aplicación de la terapia de música, así como realizar estudios de tipo cualitativo con la aplicación de esta terapia, para conocer la experiencia del participante en cuanto a sus efectos.

En cuanto a la Facultad de Enfermería, deben continuar fomentando la formación de estudiantes y docentes en el manejo de terapias y en salud mental.

\section{BIBLIOGRAFÍA}

1. González E, Silva L. Factores que ocasionan estrés en el personal de enfermería. Revista Desarrollo Científico en Enfermería. 2003; 11 (9): 279-82.

2. Fernández E, Jiménez M, Martín M. Psicología. Vol. I. Emoción y Motivación. La adaptación humana. Madrid: Ed. Centro de Estudios Ramón Areces; 2003. p. 277-79. Disponible en: http://books. google.com.mx/books?id=VwgX4NdzhU0C\&pg=PA307\&lpg=PA $307 \& \mathrm{dq}=$ Escalas + para + medir+ansiedad+normal\&source $=$ bl\&ots =j_GSyE2etb\&sig=qOq6N5YLE6n_gnAEKOaJzaMoK9s\&hl=es\&ei $=\mathrm{uJrRSanDD} 5 \mathrm{G} 6 \mathrm{tQP} 0 \mathrm{rtzJAw} \& \mathrm{sa}=\mathrm{X} \&$ oi=book_result\&resnum $=4 \& \mathrm{ct}$ $=$ result\#PPA297,M1

3. Botura A, Carvalho D, Trevisani F, Marlene. Situacoes generadoras de ansiedade e estratégias para seu controle enfermeiras: estudio preliminar. Revista Latino-Americana de Enfermagem 2003; 11 (5): (8 páginas en pantalla). Disponible en: http://www.scielo.br/scielo.php'script=sci_ arttext\&pid=S0104-11692003000500004\&Ing

4. Cano-Vindel A. Epidemiología de los trastornos de Ansiedad. Sociedad Española para el Estudio de Ansiedad y Estrés.National Institute of Mental Health.Facultad de Psicología. Universidad Complutense de Madrid, España 2012. Disponible en: http://pendientedemigracion.ucm. es/info/seas/ta/epidemi/index.htm

5. Guía Práctica. Manejo del espectro depresión-ansiedad. México: Consenso; 2004. pp. 29-60.

6. Piña-Hernández CE. Síndrome de Burnout y el desempeño laboral del personal de enfermería de una clínica psiquiátrica. Tesis de Maestría. Facultad Autonónoma de San Luis Potosí. México, 2005.

7. García M, Azpiroz J, De la Fuente Y, García M, González B. La ansiedad en el personal sanitario. Enfermería en cardiología 2007; 3 
(1): 41-45. Disponible en: http://www.enfermeraencardiologia.com/ revista/4307.pdf

8. Lara M, Medina-Mora M, Borges G, Zambrano J. Social cost of mental disorders: Disability and work days lost. Results from the Mexican survey of psychiatric epidemiology. Salud Mental. 2007; 30 (5): 4-11.

9. Valencia M. Trastornos mentales y problemas de salud mental. Día Mundial de la Salud Mental, 2007. Salud Mental. 2007; 30 (2): 75-79.

10. O’Brien P, Kennedy W, Ballard K. Enfermería Psiquiátrica. México, D.F: McGraw-Hill Interamericana; 2001. pp. 27, 339-348.

11. Cook J, Lee F. Fundamentos esenciales de enfermería en Salud Mental. 2da ed. Madrid: McGraw Hill Interamericana; 1993. pp. 27, 302-81.

12. Novel G, LLuch M, Miguel M. Enfermería Psicosocial y Salud Mental. Barcelona, España: Masson; 2005. pp. 165-70.

13. Suzanne B. Music Therapist's handbook. St. Louis: Warren H. Green, Inc.; 1987.

14. Zárate P, Díaz V. Aplicaciones de la Musicoterapia en la medicina. Rev Med Chile. 2001; 129 (2): (7 páginas en pantalla). Disponible en: http://www. scielo.cl/scielo.php?pid=S0034-98872001000200015\&script=sci_arttext

15. Wikipedia, la enciclopedia libre. Musicoterapia. (13 páginas en pantalla). Disponible en: http://es.wikipwdia.org/wiki/Musicoterapia

16. Andrews T. Sacred Sounds Magic \& Healing Through Words \& Music. United States of America: Llewellyng Publications, Woodbury, Minnesota, 2005. pp. 29-47.

17. Betés de T(Comp.). Fundamentos de Musicoterapia. Madrid: Ed. Morata; 2000. Disponible en: http://books.google.com.mx/books?id=V-eHTrxtl UYC\&pg=PA362\&lpg=PA362\&dq=m\%C3\%A9todo+Bonny+de+musi coterapia\&source $=$ bl\&ots $=$ oyzi $1 \mathrm{w} 4 \mathrm{Wr} \&$ sig $=1 \mathrm{XwLgj}$-eb9xeHaoqKchGtiQIho\&hl=es\&ei=xE2ZSse2LYbOsQP4y-ShAg\&sa=X\&oi=book_re sult \&ct=result \& resnum $=5 \# \mathrm{v}=$ onepage $\& \mathrm{q}=\mathrm{m} \% \mathrm{C} 3 \% \mathrm{~A} 9$ todo $\% 20$ Bonny $\% 20 \mathrm{de} \% 20$ musicoterapia\&f false

18. Correa I, Guedelha D. Utilización de la música en busca de la asistencia humanizada en el hospital. Universidad de Antioquia, Facultad de Enfermería. Invest Educ Enferm. 2009; 27 (1): 46-53.

19. Balarín C. Musicoterapia, Terapia del Sonido. Disponible en: http://www. enbuenasmanos.com/articulos/muestra.asp?art=1776

20. Aparicio $\mathrm{M}$ et al. ¿Cómo influye la musicoterapia en la ansiedad del paciente médico-quirúrgico sometido a una operación en el postoperatorio inmediato? Enfermería Global. 2004; 5: (4 páginas en panta- 1la). Disponible en: http://www.um.es/ojs/index.php/eglobal/article/ view/539/559

21. Flores-Gutiérrez E, Díaz J. La respuesta emocional a la música: atribución de términos de la emoción a segmentos musicales. Salud Mental. 2009; 32 (1): 21-34.

22. Mella-Mejias C, García-Alvarez R. Ansiedad en el Paciente de Consulta Externa. Acta Médica República Dominicana 1989; NoviembreDiciembre 11 (6): 212-16. Disponible en: http://bvsdo. intec.edu.do:8080/ revistas/amd/1989/11/06/AMD-1989-11-06-212-216. pdf

23. Escala Autoaplicada para la Evaluación de la Ansiedad (Zung, 1971). Disponible en: http://www.testdeansiedad.com.ar/test/escaladezung.php

24. De la Ossa S, Martínez Y, Herazo E, Campo A. Estudio de la consistencia interna y estructura factorial de tres versiones de la escala de Zung para ansiedad. Colombia Médica. 2009; 40 (1): (7 páginas en pantalla). Disponible en: http://colombiamedica.univalle.edu.co/Vol40No1/html/v40n1a6.html

25. Quiles MJ, van-der Hofstadt C J, Quiles Y. Instrumento de Evaluación del dolor en pacientes pediátricos. Rev Soc Esp Dolor 2004; 11: 360-369. Disponible en: http://scielo.isciii.es/pdf/dolor/v11n6/revision1.pdf

26. Esquema de la Escala Visual Análoga de Ansiedad. Disponible en: http:// scielo.isciii.es/pdf/dolor/v11n6/revision1.pdf

27. Stein J. Manual clínico de los Trastornos de Ansiedad. Barcelona, España: Ed. Ars Médica; 2005. pp. 3-5.

28. Verde E, Ortíz L, Monroy A, Valle I. Equidad y Salario: un estudio de Mujeres Enfermeras. Desarrollo Científ Enferm. 2004; 12 (10): 293-98.

29. Valderrama R, Castelán G, Rivera A. Diferencias entre mujeres y hombres al escuchar música sedante en el nivel del estado de ansiedad. Facultad de psicología BUAP. 2007; 11 (2): (14 páginas en pantalla). Disponible en: www.psiquiatría.com

30. García-Viniegras C, Rodríguez J, Barbón D, Cárdenas N. Musicoterapia, una modalidad terapéutica para el estrés laboral. Rev Cubana Med Gen Integr. 1997; 13 (6): (6 hojas en pantalla). Disponible en: http://scielo.sld.cu/scielo.php?script=sci_arttex\&pid $=$ S0864-21251997000600003

31. Conxa-Trallero F. Tratamiento del estrés docente y prevención del Burnout con musicoterapia autorrealizadora, Universidad de Barcelona, España, 2009. Departamento de Didáctica de la Expresión Musical y Corporal 2009: 1-32. Disponible en: http://www.musicoterapia-autorrealizadodora.net 